\title{
DATA QUALITY IN MARKETING RESEARCH - HOW TO DETERMINE ACCURACY IN ONLINE QUESTIONNAIRES
}

Boris Toma, University of Mannheim, Germany Daniel Heinrich, University of Mannheim, Germany Hans H. Bauer, University of Mannheim, Germany

\begin{abstract}
Due to its virtually unlimited technical possibilities, the Internet offers tremendous potential for the development and application of new survey methods in online market research. Thus, online questionnaires have become increasingly important. Nowadays in market research practice almost every third survey takes place on the Internet.

The range of online surveys runs from self-selection surveys in virtual communities and list-based e-mail surveys to longitudinal studies in pre-recruited online panels. Given the high degree of anonymity on the Web, however, all forms of data collection are permanently exposed to a crisis of legitimation.

Solely through an uncompromising focus on quality, this problem can be addressed. For this purpose basic methods research is required, which reveals the impact of online surveys on the quality of the data collected. Although initial research efforts have been made in this area in recent years, central design variables of online market research remain unconsidered.

Hence, in an experimental study this paper analyzes both the influence of different types of recruitment and the effects of monetary incentives on data quality. Another research goal is to develop new methods for determining the quality of data, as the recent first generation approaches of operationalizing data quality are merely unsatisfactory.
\end{abstract}

As a result of this study, innovative measurement approaches are presented and empirically validated regarding their usefulness. Using the methods of the first and second generation surprising results are shown and implications and recommendations for different stakeholders of online market research are given.

References available on request. 